\title{
Machine Learning for Modulation Classification of Radar Signals: A Survey
}

\author{
Hitham Alshoubaki
}

\begin{abstract}
Automatic modulation recognition of radar waveform is a major topic and has many military applications. This paper surveys the models and the techniques used in recognizing different modulation types of intercepted radar waveform. The literature shows the outstanding performance of deep learning neural network at low SNR values and in signaloverlapped environments as well. Additionally, using different mathematical and statistical algorithms demonstrated that utilized in features extraction of the data in order to feed them into the neural network improves the performance significantly. However, reducing computation complexity is in development too.
\end{abstract}

Keywords-Modulation classification, radar signals, deep learning, machine learning, CNN.

\section{INTRODUCTION}

Starting from 1958, it was the first use of perceptron's and a simple activation function using weights in order to gives a probabilistic model [1]. Nineteen years later, it was the first publication of back-propagation that used gradient feature of a loss function in to adjust neurons' weights of a neural network [2]. However, neural network is the main concept of Machien Learning (ML) which it has a dramatic development in variety areas; such as, image processing, video analytics, autonomous vehicles and voice recognition. Recent developments have been conducted in signal classification with very promising results. However, automatic waveform of intercepted radar signal is very important topic, especially in the modern military applications such as electronic warfare $(\mathrm{EW})$.

\section{LITERATURE REVIEW}

\section{A. Using MLP and Feature Extraction}

Automatic recognition topic is important in many modern wireless applications; and radar is not an exception. Thus, many techniques have been proposed in the literature. A good example is the supervised system utilizing Multi-Layer Perceptron (MPL) network that examined in [3], the parallel MPL network was fed by feature extracted data from intercepted radar signals; the features were extracted into time-frequency distributions by using the method of Wigner and Choi-Williams. The types of intercepted radar signals includes eight: poly-pase codes (P1, P2, P3, P4 and Frank codes), linear frequency modulation (LFM), binary phase, and discrete frequency codes (Costas codes). This approach has reach to high accuracy of the technique (98\%) at SNR's value of $6 \mathrm{~dB}$.

\section{B. Using CNN and Deep Q-Learning Nerwork}

Due to the increasing complexity environment of the radar waveforms and complexity of analyze radar signal that constructed from multiple component, a framework in [4] was proposed that can recognize different types of any overlapping radar signals, whether it is a two component radar waveform or only one component radar waveform. A CNN deep network besides a Deep Q-Learning Network (DQN) approach successes to classify different types of radar intercepted waveforms includes Frank code, linear frequency modulation (LFM), BPSK, Frequency Shift Key (2FSK, 4FSK and EQFM), SFM and MP. The probability success recognition (PSR) reached to around $94 \%$ at SNR value of $-6 \mathrm{~dB}$. However, it needs high number of the training data set to have high efficiency.

\section{Using CNN and Multi-Instance Multi-Label Learning}

The high density of electromagnetic environment is a challenge for the low probability intercept (LPI) radars as they receive overlapped signals considering that their signals being transmitted concurrently on same band. In [5], a framework of a Multi-Instance Multi-Label Learning (MIML) technique utilized to works along with a Convolutional Neural Network (CNN). The approach have been developed in order to classify each single radar signal in an overlapped environment. Following four modulations have been test: Costas codes, Barker codes, linear frequency modulation (LFM) and Frank codes. The approach able to identifies the received signals in different SNR values (-10 dB, $6 \mathrm{~dB}$ and $10 \mathrm{~dB})$. However, the network has been trained utilizing only a single type of radar signals.

\section{Using CNN and Sample Averaging Technique}

In [6], another approach that proposed to detect and recognize LPI radar signal. The hyper-parameters (number inputs, number of neurons, number of filters and the filters' sizes) of CNN have been determined according modulation kind of the radar waveform which in order to obtain highest performance. The results shows that applying Sample Averaging Technique (SAT) could decrease the number of samples remarkably which consequence increasing the recognition performance dramatically; keeping the same computation cost in the same time. However, the used signal waveform modulations in the examination are poly-pase codes (P1, P2, P3, P4 and Frank codes), BFSK, no modulation and LFM.

\section{E. Using CNN and Time-frequency Images Technique}

The changing in the frequency with the time is a one of the fundamental distinction of the radar signals in different kinds of modulation. This feature has been well used in [7] by converting a one-dimensional radar signal to time-frequency images (TFIs); then utilizing analysis of the time-frequency and analysis of statistical characteristics signals' noise into a deep CNN network. Besides the high recognition rate at low 
SNR levels, the results illustrates substantial generalization of the framework. However, dataset consists of three modulation types: LFM, SCR, and PCR3.

\section{F. Using Fractional Fourier Transform (FRT)}

Away from machine learning methods, automatic modulation classification of LPI radar waveform could be obtain by other methods as highlighted in [8]. The approach of classification was based on the features extraction of the intercepted radar waveform by measuring fractional Fourier transform (FRT) utilizing frequency-domain and time-domain and method. The types of radar signal modulation includes poly-phase (Frank codes and P1 - P4 codes), linear frequency modulation (LFM), poly-phase Barker, binary frequency shift keying (BFSK) and nonlinear linear frequency modulation. The results indicates high correctness recognition rate $(95 \%)$ at stubby SNR values. Furthermore, the algorithm need lower computational resources than other typical methods i.e. WVD.

\section{G. Using SSD and Supplementary Classifier}

Although LPI radars are more common used than pulse waves (PW) due to the low power peak of the LPI radar signals, but there is a few papers studied recognition of continuous wave $(\mathrm{CW})$. In [9], the proposed framework to have automatic recognition for CW LPI and PW radar signals. The used methods depends on single shot multiple box detector (SSD) besides an additional supplementary classifier. A 12 waveforms modulation are used in the experiment: Costas codes, Binary Phase Shift Key (BPSK), poly-phase (P1 - P4 codes and Frank codes), Linear Frequency Modulation (LFM), and $\mathrm{T} 1$ - T4 codes. The results shows that the proposed approach have similar high recognition performance without the necessity of knowing the first and last samples points of the intercepted radar signals. The results proves the used method has superior recognition performance when applying it on CW LPI radar signals.

\section{H. Using ENN and Features Extraction}

The approach that have been followed in [10] in order to automatically recognize the modulation of the received LPI radar waveform is utilizing Elman neural network (ENN). Additionally, several features extraction techniques; includes principal component analysis (PCA), Pseudo-Zernike moments (PZM), binarization algorithm (BA), etc. However, extraction of the features is though using Choi-Williams 2D time-frequency distribution (CWD) of intercepted radar signal. For simplicity, redundant features can be neglected through an algorithm of features selection, which divides the features into two classes. First class is (Signal Features) can be extracted directly from the radar waveform; the second class called Image Feature, which obtains from the timefrequency image. The test waveform modulations are: polyphase (P1, P2, P3, P4 and Frank codes), linear frequency modulation (LFM), binary phase shift key (Barker modulation) and Costas codes. The simulation indicates a great automatic classification performance of the proposed framework, which reach to $94.7 \%$ correct recognition rate at SNR value of $-2 \mathrm{~dB}$.

\section{Using CNN, CWD and SVM}

Recognition Rate of LPI radar signal is a challenge, authors in [11] proposed model that could address this topic. The proposed model has three main parts. It begins by applying Choi-Williams distribution (CWD) to convert an intercepted LPI radar waveform to time-frequency image. After that, feed the image into two pre-trained deep convolutional network (Inception-v3 and ResNet-152) for features extraction. Then, utilize the output into the classifier, which it is a support vector machine (SVM) model. Types of waveform modulation are binary frequency shift keying (BFSK), Frank modulation, T1 - T2 codes, Costas modulation and linear frequency modulation (LFM). The results shows that successful recognition rate at SNR value of $-2 \mathrm{~dB}$ was reached to $96.2 \%$ for using Inception-v3; on the other hand, ResNet-152 reached to $97.8 \%$ at same SNR value.

\section{J. Using CNN, ENN and CWD}

A hybrid classifier model is proposed in [12] in order to classify the LPI radar intercepted waveform automatically. A combination of deep Elman neural network (ENN) besides the other well-defined deep convolution neural network (CNN) and the key extracted features can be obtained as image into 2D in the time-frequency distribution by utilizing ChoiWilliams (CWD). However, 12 types of waveform modulation used in the testing i.e. poly-phase (Frank codes, P1, P2, P3 and P4 codes), Barker modulation of Binary phase shift keying (BPSK), Costas codes, linear frequency modulation (LFM) and (T1, T2 T3 and T4 codes). The simulation results illustrate high average accuracy rate in classification $(94.5 \%)$ which could be achieved at SNR value of $-2 \mathrm{~dB}$.

\section{CRITICAL ANALYSIS AND FUTURE WORK}

The literature shows that the deep learning techniques, especially $\mathrm{CCN}$, have promising results in the recognition of radar waveforms, but the limitation of having data set of radar signal waveform is a big challenge; in order to overcome this challenge, other techniques may utilized besides $\mathrm{CNN}$ such as MIML which requires less waveforms to train the $\mathrm{CNN}$ network. However, other techniques may added to CCN such as CWD; this combination increases the recognition accuracy rate considerably. Another important aspect is the generalization capability, using TFI and CNN together showed significant generalization. Computation cost is nevertheless important, which can be reduced dramatically by reducing the space diminution of the radar waveform using SAT before fed it into the CNN. Table I summaries the different techniques, and features extractions methods that used besides deep convolutional neural networks and highlights the advantage and the disadvantage of each combination. However, verity of radar waveform modulation types are shown in the table for the comparison as well. Still above aspects are subjective for improving; additionally, reducing the noise of radar signal before the process would be the future work.

TABLE I. RECOGNITION METHODS USING CNN BESIDES Other TECHNIQUES

\begin{tabular}{|l|l|}
\hline \multicolumn{1}{|c|}{ Model of Recognition } & Advantages / Disadvantages \\
\hline \multicolumn{1}{|c|}{ CNN + DQN } & \\
Intra-pulse modulation of overlapping & \\
single/dual component of radar & - High accuracy. \\
waveform includes: & \\
- Frank code. & - Requires a large number of \\
- Linear frequency modulation (LFM). & data sets to be educated \\
- BPSK. & \\
- Frequency Shift Key (2FSK, 4FSK & \\
and EQFM). & \\
- SFM. & \\
- MP.
\end{tabular}




\begin{tabular}{|c|c|}
\hline Model of Recognition & Advantages / Disadvantages \\
\hline $\begin{array}{l}\text { CNN + MIML } \\
\text { Overlapping LPI Radar Waveforms } \\
\text { includes: } \\
\text { - Costas codes. } \\
\text { - Barker codes. } \\
\text { - Linear frequency modulation (LFM). } \\
\text { - Frank codes. }\end{array}$ & $\begin{array}{l}\text { Only one form of single } \\
\text { was used during training }\end{array}$ \\
\hline $\begin{array}{l}\quad \text { CNN + SAT } \\
\text { LPI Radar Waveform includes: } \\
\text { - No modulation } \\
\text { - BFSK. } \\
\text { - LFM. } \\
\text { - Frank. } \\
\text { - (P1 - P4). }\end{array}$ & $\begin{array}{l}\text { - High accuracy. } \\
\text { - Reducing Computation } \\
\text { Complexity }\end{array}$ \\
\hline $\begin{array}{l}\qquad \text { CNN + TFI } \\
\text { Radar Waveforms includes: } \\
\text { - LFM. } \\
\text { - SCR. } \\
\text { - PCR3. }\end{array}$ & $\begin{array}{l}\text { - High accuracy. } \\
\text { - Strong generalization. }\end{array}$ \\
\hline $\begin{array}{l}\quad \text { CNN + CWD + SVM } \\
\text { LPI Radar Waveform includes: } \\
\text { - Binary frequency shift keying } \\
\text { (BFSK). } \\
\text { - Frank modulation. } \\
\text { - (T1 - T2) codes. } \\
\text { - Costas modulation. } \\
\text { - Linear frequency modulation (LFM). }\end{array}$ & - High accuracy. \\
\hline $\begin{array}{l}\quad \text { CNN + ENN + CWD } \\
\text { LPI Radar Waveform includes: } \\
\text { - Poly-phase (Frank codes, P1, P2, P3 } \\
\text { and P4 codes), } \\
\text { - Barker modulation of binary phase } \\
\text { shift keying (BPSK), } \\
\text { - Costas codes, } \\
\text { - Linear frequency modulation (LFM) } \\
\text { - (T1, T2 T3 and T4) codes. }\end{array}$ & - High accuracy. \\
\hline
\end{tabular}

On the other hand, table II summaries the many other techniques and features extractions methods that without utilizing deep convolutional neural networks, instead, other techniques that includes the conventional MLP network, CWD, FRT, SSD and other supplementary classifier are used; the table highlights the advantage and the disadvantage of each combination. However, verity of radar waveform modulation types are shown in the table for the comparison as well.

TABLE II. RECOGNITION METHODS WITHOUT USING CNN

\begin{tabular}{|c|c|}
\hline Model of Recognition & Advantages / Disadvantages \\
\hline $\begin{array}{l}\qquad \text { MLP + CWD } \\
\text { Radar waveforms includes: } \\
\text { - Poly-pase codes (P1, P2, P3, P4 and } \\
\text { Frank codes). } \\
\text { - linear frequency modulation (LFM) } \\
\text { - Binary phase (BPSK). } \\
\text { - Discrete frequency codes (Costas } \\
\text { codes). }\end{array}$ & $\begin{array}{l}\text { - A good correct } \\
\text { classification for various } \\
\text { types of waveforms. }\end{array}$ \\
\hline 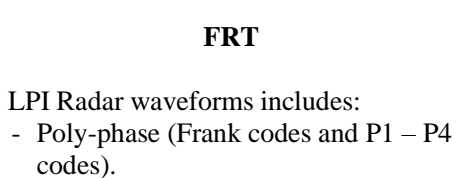 & $\begin{array}{l}\text { - Differentiating between } \\
\text { various forms of } \\
\text { modulations with high } \\
\text { success rate at a low SNR. }\end{array}$ \\
\hline
\end{tabular}

\begin{tabular}{|c|c|}
\hline Model of Recognition & Advantages / Disadvantages \\
\hline $\begin{array}{l}\text { - Linear frequency modulation (LFM). } \\
\text { - Poly-phase Barker. } \\
\text { - Binary frequency shift keying } \\
\text { (BFSK). } \\
\text { - Nonlinear linear frequency } \\
\text { modulation. }\end{array}$ & $\begin{array}{l}\text { - Less computational } \\
\text { complexity. }\end{array}$ \\
\hline $\begin{array}{l}\text { SSD + Supplementary Classifier } \\
\text { PW and CW LPI radar waveforms } \\
\text { includes: } \\
\text { - Costas codes, } \\
\text { - Binary Phase Shift Key (BPSK), } \\
\text { - Poly-phase (P1 - P4 codes and Frank } \\
\text { codes), } \\
\text { - Linear Frequency Modulation (LFM) } \\
\text { - (T1 - T4) codes. }\end{array}$ & $\begin{array}{l}\text { - Extraordinary recognition } \\
\text { performance of CW-LPI } \\
\text { waveform. } \\
\text { - Without the condition of } \\
\text { prior knowing of the } \\
\text { starting and last samples of } \\
\text { PW waveform. }\end{array}$ \\
\hline $\begin{array}{l}\quad \text { ENN + CWD + PCA } \\
\text { LPI Radar Waveform includes: } \\
\text { - Poly-phase (P1, P2, P3, P4 and Frank } \\
\text { codes), } \\
\text { - Linear frequency modulation (LFM). } \\
\text { - Binary phase shift key (Barker } \\
\text { modulation). } \\
\text { - Costas codes. }\end{array}$ & - High accuracy. \\
\hline
\end{tabular}

\section{CONCLUSION}

Applications of automatic modulation classification for radar signal is oriented to military field in the last decade. This paper provides a literature review of the models and the techniques used to recognize the modulation of intercepted radar waveform. It shows the significant performance of deep learning neural network and CDW at low SNR values and in signal-overlapped environments as well. Training data set can be reduced via MIML with $\mathrm{CNN}$, having a good generalization can obtained by utilizing TFI besides CNN. On the other hand, other machine learning models without utilizing CNN shows a decent results as well, i.e. MPL and CWD, FRDT and SSD that take advantage of the mathematical and statistical algorithms in the features extraction process of the intercepted radar waveform makes the performance more efficient. Although the promising testing results of $\mathrm{CNN}$ and other machine learning methods, these models still need to enhance their accuracy, the required training data set size and computation running time in the future work.

\section{REFERENCES}

[1] Rosenblatt, Frank. The perceptron: a probabilistic model for information storage and organization in the brain. Psychological review, 65(6):386, 1958 .

[2] Werbos, Paul. Beyond regression: New tools for prediction and analysis in the behavioral sciences. 1974.

[3] J. Lundén and V. Koivunen, "Automatic Radar Waveform Recognition”, IEEE Journak of Selected Topics in Signal Processing, Vol. 1, No. 1, June 2007.

[4] Z. Qu , C. Hou , C. Hou, W. Wang, "Radar Signal Intra-Pulse Modulation Recognition Based on Convolutional Neural Network and Deep Q-Learning Network", IEEE Access, Vol. 8, 2020.

[5] Z. Pan , S. Wang, M. Zhu , S. and Y. Li, "Automatic Waveform Recognition of Overlapping LPI Radar Signals Based on MultiInstance Multi-Label Learning”, IEEE Signal Processing Letters, Vol. 27, 2020

[6] S. Kong, I. Kim, L. Hoang and E. Kim, "Automatic LPI Radar Waveform Recognition Using CNN", IEEE Access, Vol. 6, January 2018 . 
[7] C. Wang, J. Wang and X. Zhang, "Automatic Radar Waveform Recognition Based on Time-frequency Analysis and Convolutional Neural Network", IEEE International Conference on Acoustics, Speech and Signal Processing (ICASSP), 2017.

[8] T. Kishore, K. Deergha Rao, "Automatic Intrapulse Modulation Classification of Advanced LPI Radar Waveforms", IEEE Transactions on Aerospace and Electronic Systems, Vol. 53, Issue 2, April, 2017.

[9] L. Hoang , M. Kim and S. Kong, "Automatic Recognition of General LPI Radar Waveform Using SSD and Supplementary Classifier", IEEE Transactions on Signal Processing, Vol. 67, No. 13, July, 2019.
[10] M. Zhang, L. Liu, and M. Diao, "LPI radar waveform recognition based on time-frequency distribution," Sensors, vol. 16, no. 10, 2016, Art. no. 1682.

[11] Q. Guo, X. Yu, and G. Ruan, "LPI radar waveform recognition based on deep convolutional neural network transfer learning," Symmetry, vol. 11, no. 4, pp. 540.1-540.14, 2019.

[12] Ming, Z.; Ming, D.; Lipeng, G.; Lutao, L. "Neural Networks for Radar Waveform Recognition", Symmetry Basel 2017, 9, 75. 